\title{
(6) OPEN ACCESS \\ Positive effects on bone mineralisation and muscular fitness after 10 months of intense school-based physical training for children aged 8-10 years: the FIT FIRST randomised controlled trial
}

\author{
Malte Nejst Larsen, ${ }^{1}$ Claus Malta Nielsen, ${ }^{1,2}$ Eva Wulff Helge, ${ }^{1}$ Mads Madsen, ${ }^{1}$ \\ Vibeke Manniche, ${ }^{2}$ Lone Hansen, ${ }^{3}$ Peter Riis Hansen, ${ }^{4}$ Jens Bangsbo, ${ }^{1}$ \\ Peter Krustrup ${ }^{1,5}$
}

${ }^{1}$ Department of Nutrition, Exercise and Sports, Copenhagen Centre for Team Sport and Health, University of Copenhagen, Copenhagen, Denmark

${ }^{2}$ Frederikssund Municipality, Frederikssund, Denmark ${ }^{3}$ Team Danmark, Brøndby, Denmark

${ }^{4}$ Department of Cardiology, Herlev-Gentofte University Hospital, Hellerup, Denmark ${ }^{5}$ Department of Sport and Health Sciences, College of Life and Environmental Sciences, University of Exeter, Exeter, UK

\section{Correspondence to} Professor Peter Krustrup, Department of Nutrition, Exercise and Sports, Copenhagen Centre for Team Sport and Health, University of Copenhagen, Copenhagen, Denmark, Universitetsparken 13, Copenhagen DK-2100, Denmark:

pkrustrup@nexs.ku.dk

Accepted 1 May 2016 Published Online First 13 June 2016

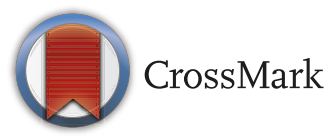

To cite: Larsen $\mathrm{MN}$, Nielsen CM, Helge EW, et al. Br I Sports Med 2018:52:254-260.

\section{ABSTRACT \\ Objectives We investigated whether musculoskeletal fitness of school children aged 8-10 years was affected by frequent intense PE sessions.}

Design and participants 295 Danish school children aged 8-10 years were cluster randomised to a small-sided ball game group (SSG) ( $n=96$, four schools, five classes), a circuit strength training group (CST) $(n=83$, four schools, four classes) or a control group (CON, $n=116$, two schools, five classes).

Intervention SSG or CST was performed $3 \times 40$ min/week over 10 months. Whole-body dual-energy $X$-ray absorptiometry (DXA) scans were used to determine areal bone mineral density (aBMD), bone mineral content (BMC) and lean body mass (LBM). Flamingo balance, standing long jump and 20-m sprint tests were used to determine muscular fitness.

Results Analysis of baseline-to- 10 months change scores showed between-group differences in favour of the interventions in whole-body aBMD (SSG vs CON: $8 \mathrm{mg} /$ $\mathrm{cm}^{2}, 95 \% \mathrm{Cl} 3$ to $13 ;$ CST vs CON: $7 \mathrm{mg} / \mathrm{cm}^{2}, 95 \% \mathrm{Cl} 2$ to $13, \mathrm{p}<0.05$ ) and leg BMC (SSG vs CON: $11 \mathrm{~g}, 95 \% \mathrm{Cl}$ 4 to 18 ; CST vs CON: $11 \mathrm{~g}, 95 \% \mathrm{Cl} 3$ to $18, \mathrm{p}<0.05$ ). SSG had higher change scores in leg aBMD compared with CON and CST (SSG vs CON: $19 \mathrm{mg} / \mathrm{cm}^{2}, 95 \% \mathrm{Cl} 11$ to $39, p<0.05$; SSG vs CST: $12 \mathrm{mg} / \mathrm{cm}^{2}, 95 \% \mathrm{Cl} 3$ to 21 , $\mathrm{p}<0.05)$, and CST had higher change scores in whole-body BMC compared with CON (CST vs CON: $25 \mathrm{~g}, 95 \% \mathrm{Cl} 10$ to $39, p<0.05)$. Both training types resulted in higher change scores in postural balance (SSG vs CON: 2.4 fewer falls/min, $95 \%$ Cl 0.3 to 4.5, CST vs CON: 3.6 fewer falls/ min, $95 \% \mathrm{Cl} 1.3$ to $5.9, \mathrm{p}<0.05$ ) and jump length (SSG vs CON: $10 \%, 95 \%$ Cl 5 to $16 \%$; CST vs CON: $9 \%, 95 \%$ Cl 3 to $15 \%, p<0.05)$. No between-group differences were observed for sprint performance or LBM ( $p>0.05)$.

Conclusions In conclusion, $3 \times 40$ min/week with SSG or CST over a full school year improves bone mineralisation and several aspects of muscular fitness of children aged 810 years, suggesting that well-organised intense physical education classes can contribute positively to develop musculoskeletal health in young children.

Trial registration number NCT02000492, post results.

\section{INTRODUCTION}

The incidence of osteoporotic fractures is increasing in the ageing population ${ }^{1}$ and the fracture risk relates, among other things, to bone strength, muscle strength and postural balance. ${ }^{2}$ It has been shown that weight-bearing activities during childhood have a positive effect on variables related to bone strength ${ }^{34}$ and that low bone strength in childhood is associated with a higher fracture risk in later life. ${ }^{5}$ This indicates that physical activity in childhood leads to residual benefits in bone density and strength in adulthood (tracking); ${ }^{2}{ }^{6}$ thus, it is of great importance to investigate the osteogenic impact of different weight-bearing training types during childhood that may have the potential to decrease the risk of osteoporosis in later life. The school setting is considered a favourable platform for promoting a physically active lifestyle, as there is a possibility of reaching all children and most importantly those who have low fitness levels and impaired musculoskeletal health, including low bone mineral density (BMD). Special attention should be given to girls with limited physical activity in leisure time and low calcium intake, ${ }^{7-9}$ who therefore miss the opportunity to optimise bone mass in the peripubertal years that are characterised by a high responsiveness to osteogenic bone loading activity. ${ }^{5}$ School-based exercise could preferably be integrated in the physical education (PE) lessons, and also in extracurricular physical activities. ${ }^{5}$ As the National Osteoporosis Foundation states in a position paper on the impact of lifestyle factors on the development of peak bone mass, ${ }^{5}$ there is strong evidence that physical activity in youth does have a positive impact on bone accrual and improvement of peak bone mass in the third decade of life. However, the osteogenic effect varies due to differences in the exercise activities regarding important factors such as load magnitude, load rate, load frequency and repetitiveness. ${ }^{10}$ Thus, some studies have found that schoolbased interventions involving increased volume of physical activity have positive effects on BMD in girls as well as boys, ${ }^{11-14}$ while others have found no effect on bone mineral content (BMC) and BMD in children attending sports schools compared with traditional schools. ${ }^{15}$ However, in the same study, positive effects of practising leisure-time sports were found, and this potential contradiction may be related to the phenomenon that training-induced effects are dependent on the type of activity and the intensity of training.

Several studies from our research group have demonstrated favourable cardiovascular, metabolic 
and musculoskeletal effects of football training in healthy women and men aged $20-75$ years as well as in various patient groups. ${ }^{16-18}$ Interestingly, a recent randomised controlled trial (RCT) study found a significant correlation between the average number of decelerations during a football session and the magnitude of bone effects over time, ${ }^{18}$ which may be ascribed the larger osteogenic impact associated with higher strain magnitude and rates when moving fast. Furthermore, various types of strength and high-impact jump training have been observed to have positive effects on functional capacity and bone mineralisation in healthy men and women across the lifespan. ${ }^{16} 19$ However, further studies are required in order to elucidate the extent to which these findings are applicable in children.

Recent investigations from our group have shown that the aerobic and musculoskeletal intensity is high in children during a variety of team sports (football, basketball and unihockey). ${ }^{20} 21$ It has been shown that, prior to puberty, footballers have superior bone health status compared with children not active in sports clubs as well as children active in sports clubs practising non-weight-bearing sports such as swimming and cycling. ${ }^{22-24}$ Moreover, longitudinal studies have shown that football participation throughout the pubertal years results in a twofold increase in femoral BMC and areal BMD (aBMD). ${ }^{25}{ }^{26}$ It has also been suggested that circuit strength training (CST) can enhance selected measures of lower body muscle power in children, ${ }^{27}{ }^{28}$ whereas effects of CST on childhood bone health are unclear. ${ }^{29}$ Moreover, it has not yet been investigated whether specific school-based interventions with small-sided ball game activities or CST are affecting gross motor skills in young children.

Thus, the aim of the present study was to conduct a longterm cluster-randomised controlled training intervention to investigate whether the bone mineralisation and muscular fitness of children aged 8-10 years were affected by 10 months of highintensity school-based training regimes $(3 \times 40 \mathrm{~min} /$ week $)$ consisting of odd-impact small-sided football and other ball games or circuit strength training with weight-bearing exercises.

\section{METHODS}

A total of 295 Danish third-grade school children aged 810 years from six schools (three urban and three rural schools) were cluster randomised into the SSG $(n=96$, four schools, five classes), CST ( $\mathrm{n}=83$, four schools, four classes) or control group (CON, $n=116$, two schools (one urban, one rural), five classes). The Consolidated Standards of Reporting Trials (CONSORT) 2010 flow diagram is presented as figure 1. Two rounds of block randomisation were used to assign one control school and two training intervention schools in each geographical area, and to assign the 2-3 school classes in the training intervention schools to SSG or CST, with both training types represented at all training intervention schools. Two staff members packed sets of identical, sealed, opaque envelopes with the names of the schools from the rural and urban area, and the names of the school classes, respectively, along with two sets of envelopes that contained the numbers $1-2,3-4$ and 5-6. A third researcher who was blinded to the packing of envelopes chose envelopes and rolled a dice to decide group allocation and training type, respectively.

All pre and post-tests were performed during school time in the beginning and at the end of the school year by university staff members with the support of school teachers. Child assent and written informed parental consent were obtained for all participants. The study was approved by the committees on Biomedical Research Ethics for the Capital Region of Denmark

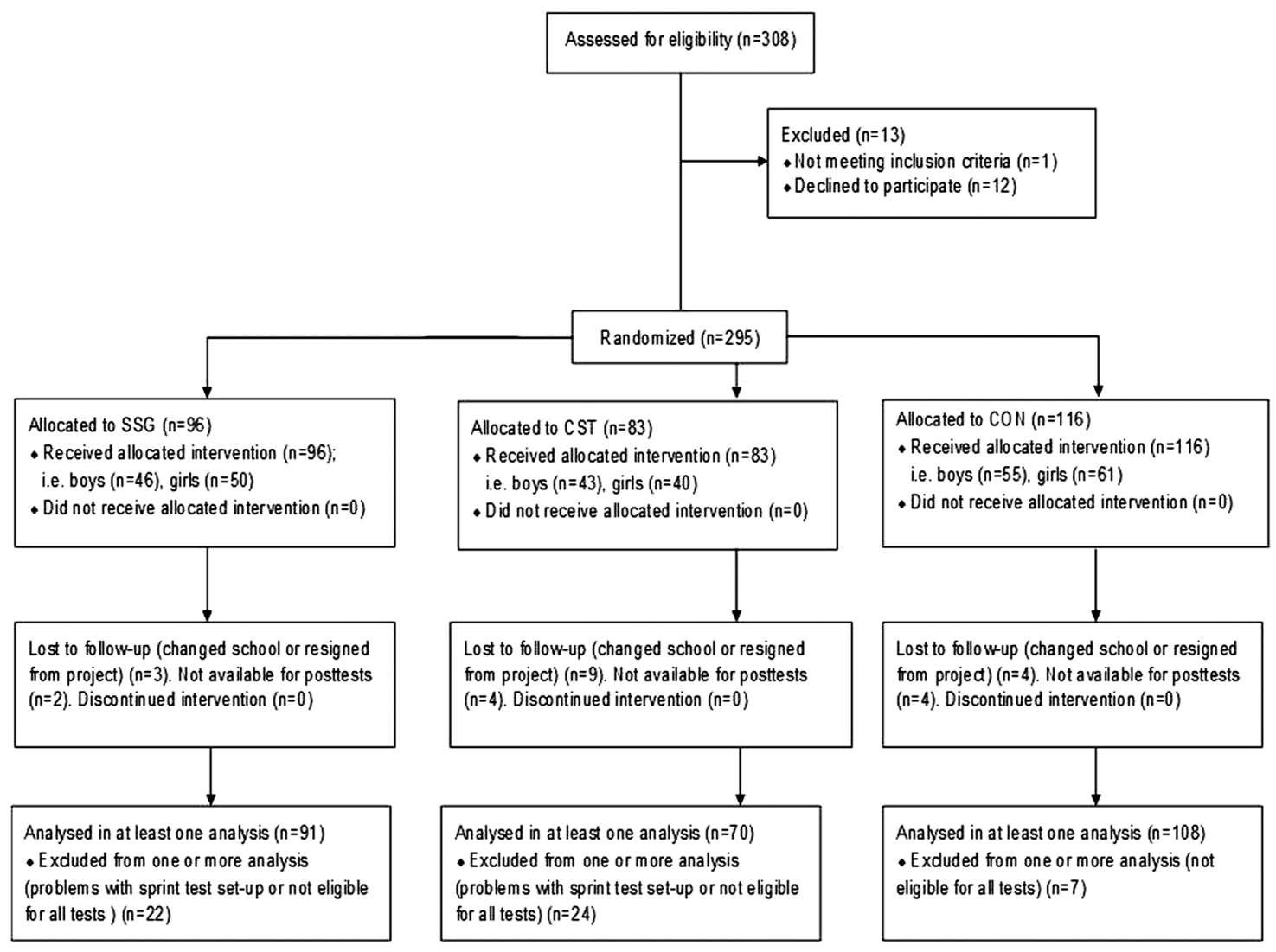

Figure 1 Participant flow chart. CONSORT, Consolidated Standards of Reporting Trials. 
(J.no. H-3-2013-038). The present study is part of the large-scale Frequent Intense Training-Football, Interval Running and Strength Training 'FIT FIRST' study with a total of >400 children (ClinicalTrials.gov: NCT02000492). The present study focuses on the effects of $3 \times 40$ min weekly (2 hours weekly) SSG and CST training versus control, whereas data for the two training groups with high-frequency, lowvolume $(5 \times 12$ min weekly) SSG and interval running will be presented in future publications.

All the children were interviewed and examined by a medical doctor who also assessed Tanner stage. One child was excluded because of physical disabilities. During the interview, the children were asked whether they participated in organised sport activities at least once per week (yes or no) (table 1).

\section{Training}

The two different types of training (SSG and CST) were led by trainers engaged part time by the university. The training sessions were performed at the schools in the afternoon, three times per week, spread over 4 or 5 days of the week. SSG consisted mainly of $3 \mathrm{v} 3$ football ( $75 \%$ of training sessions), but also $3 v 3$ basketball and unihockey (floorball). For practical reasons, some other small-sided games or team sizes (maximally $4 \mathrm{v} 4$ ) were occasionally used. Each training session began with a 35 min warm-up with drills relevant to the ball game used in that particular session.

CST consisted of $30 \mathrm{~s}$ all-out exercise periods interspersed by $45 \mathrm{~s}$ rest periods. Half the time, it was conducted as traditional circuit training with 6-10 stations with plyometric and strength exercises such as jumps, sit-ups, push-ups and other dynamic exercises as well as static strength training using upper and lower body, as well as the core. The other half consisted of wellknown games activating the children in the same way as the traditional CST. The instructors recorded attendance of participants in a training $\log$ as either present, not present or half present if someone arrived late or left early.

\section{Anthropometric measures and dual-energy X-ray absorptiometry}

Barefooted and wearing light clothing, the children were weighed (Tanita WB-110MA, Tanita, Europe) and height was measured (235 Heightronic Digital Stadiometer, QuickMedical, Issaquah, Washington, USA). On the basis of weight and height, a whole-body dual-energy X-ray absorptiometry (DXA) scan (Lunar Prodigy; E Medical Systems, Madison, Wisconsin, USA) using Encore software V.13.5 (Encore, Madison, Wisconsin, USA) was performed in appropriate mode (thin, thick or normal) for estimation of whole-body and leg aBMD and BMC as well as lean body mass (LBM). The manufacturer states a coefficient of variation (CV) for whole-body bone variables of $<1 \%$. This is supported by Margulies et al ${ }^{30}$ who also evaluated the leg region in children from a whole-body scan and report CV values of $1.11-1.36 \%$ for the regional (leg) bone parameters. For the individual child, pretesting and post-testing were conducted at the same time of the day and the children were instructed to fast for at least 2 hours and to visit the toilet prior to the scan. The children were scanned in a supine position.

\section{Postural balance}

Postural balance was evaluated using the single-legged Flamingo balance test ${ }^{31}$ performed on a $3 \mathrm{~cm}$ wide, $5 \mathrm{~cm}$ high and $50 \mathrm{~cm}$ long balance beam. The participant was instructed to stand barefooted on one leg on the bar, with eyes open, holding the contralateral leg at the ankle joint. The number of times the participant fell off the bar (defined as touching the ground or/and unable to hold the contralateral leg at the ankle joint) within

Table 1 Participants' characteristics and sports club involvement in relation to group (SSG, CST and CON) and gender before (pre) and after (post) the 10-month intervention period

\begin{tabular}{|c|c|c|c|c|c|c|}
\hline & \multicolumn{2}{|l|}{ SSG } & \multicolumn{2}{|l|}{ CST } & \multicolumn{2}{|l|}{ CON } \\
\hline & Pre & Post & Pre & Post & Pre & Post \\
\hline \multicolumn{7}{|c|}{ Age (years) } \\
\hline All & $9.3( \pm 0.4)$ & $10.1( \pm 0.4)$ & $9.3( \pm 0.3)$ & $10.0( \pm 0.3)$ & $9.3( \pm 0.3)$ & $10.0( \pm 0.4)$ \\
\hline Boys & $9.3( \pm 0.4)$ & $10.1( \pm 0.4)$ & $9.2( \pm 0.4)$ & $9.9( \pm 0.3)$ & $9.3( \pm 0.3)$ & $10.0( \pm 0.3)$ \\
\hline Girls & $9.3( \pm 0.3)$ & $10.1( \pm 0.3)$ & $9.3( \pm 0.3)$ & $10.0( \pm 0.4)$ & $9.4( \pm 0.3)$ & $10.0( \pm 0.4)$ \\
\hline \multicolumn{7}{|c|}{ Weight (kg) } \\
\hline All & $32.8( \pm 7.0)$ & $35.4( \pm 7.6)$ & $32.2( \pm 7.0)$ & $35.4( \pm 7.6)$ & $32.8( \pm 6.0)$ & $35.2( \pm 6.8)$ \\
\hline Boys & $32.6( \pm 5.4)$ & $34.9( \pm 5.5)$ & $32.2( \pm 7.3)$ & $35.8( \pm 7.4)$ & $31.7( \pm 5.2)$ & $33.8( \pm 5.1)$ \\
\hline Girls & $33.0( \pm 8.2)$ & $35.8( \pm 9.0)$ & $32.2( \pm 6.7)$ & $35.1( \pm 7.7)$ & $33.5( \pm 6.7)$ & $36.3( \pm 7.8)$ \\
\hline \multicolumn{7}{|c|}{ Height (m) } \\
\hline All & $138.4( \pm 6.8)$ & $142.3( \pm 7.3)$ & $137.3( \pm 5.7)$ & $141.5( \pm 6.0)^{*}$ & $138.4( \pm 6.0)$ & $142.2( \pm 6.4)$ \\
\hline Boys & $139.2( \pm 6.4)$ & $143.0( \pm 6.8)$ & $138.7( \pm 5.5)$ & $142.7( \pm 5.7)$ & $137.9( \pm 5.3)$ & $141.5( \pm 5.3)$ \\
\hline Girls & $137.6( \pm 7.1)$ & $141.7( \pm 7.6)$ & $136.0( \pm 5.7)$ & $140.4( \pm 6.1)$ & $138.7( \pm 6.5)$ & $142.8( \pm 7.0)$ \\
\hline \multicolumn{7}{|c|}{ Tanner stage $(1 / 2 / 3) \%$} \\
\hline All & $86 / 14 / 0$ & $77 / 23 / 0$ & $98 / 2 / 0$ & $84 / 24 / 0$ & $84 / 14 / 2$ & $73 / 27 / 0$ \\
\hline Boys & $100 / 0 / 0$ & $98 / 2 / 0$ & $100 / 0 / 0$ & $100 / 0 / 0$ & $100 / 0 / 0$ & $100 / 0 / 0$ \\
\hline Girls & $81 / 19 / 0$ & $62 / 38 / 0$ & $95 / 5 / 0$ & $59 / 41 / 0$ & $70 / 26 / 3$ & $54 / 46 / 0$ \\
\hline \multicolumn{7}{|c|}{ Sports club active (\%) } \\
\hline All & 73 & 74 & 72 & 73 & 60 & 61 \\
\hline Boys & 76 & 76 & 77 & 79 & 59 & 59 \\
\hline Girls & 71 & 72 & 68 & 67 & 61 & 59 \\
\hline
\end{tabular}

Data are presented as mean $( \pm S D)$, except for Tanner stage distribution ( $\%$ in $1 / 2 / 3$, respectively) and the percentage of sports club active children (\% of all).

${ }^{*}$ Denotes difference in change scores compared with CON, $p<0.05$.

CON, control group; CST, circuit strength training; SSG, small-sided ball game training. 
1 min of stance time was counted and used as an indicator of postural balance. ${ }^{31}$ The participants were given one try with each leg before choosing their preferred leg for the test. If 20 falls were counted before $1 \mathrm{~min}$ standing time had elapsed, the time was noted and used to calculate the expected number of falls during a full $1 \mathrm{~min}$ period. The test has been proved to be reliable and valid as a test for balance in young children. ${ }^{32}$

\section{Maximal horizontal jump length}

The maximal horizontal jump test was performed as a squat jump (SJ) with the arms held steady behind the back and from standing erect, starting with a knee flexion to the squat position which was held in $2 \mathrm{~s}$ before a maximal forward jumping and as a countermovement jump (CMJ) allowing use of the arms. After a standardised warm-up, the children were placed behind a line with their feet parallel and shoulder-width apart starting with the CMJ followed by the SJ after a 2 min rest period. In both jumps, the distance from the starting line to the heel was measured. Maximal horizontal jump length is a reliable method that is well correlated with muscular leg strength in children. ${ }^{33} 34$

\section{$20 \mathrm{~m}$ sprint test}

After the warm-up, the children performed $2 \times 20 \mathrm{~m}$ maximal sprints with at least $2 \mathrm{~min}$ of recovery between sprints. All sprints started from a standing position and were timed using two ports of light sensors (Witty Microgate, Bolzano, Italy) placed at $0 \mathrm{~m}$ (positioned $30 \mathrm{~cm}$ in front of the standing start position) and $20 \mathrm{~m}$ : the fastest time was recorded as the test result. ${ }^{35}$ Sprinting abilities rely on muscle strength, body weight and neuromuscular function.

\section{Gross motor skill}

Time (in seconds) to complete a coordination wall with three stages of increased difficulty was used to evaluate gross motor skill. $^{36}$ Each stage consisted of a table $(9 \times 8$ A5 size squares) with numbered marks from 1 to 10 . Half the numbers were blue, half were red. The two lowest ranks were separated from the higher ranks by a thick line. The participants had a red mark on the right hand and a blue mark on the left hand, and were instructed to touch the numbers from 1 to 10 in the correct order, and as fast as possible with the hand (above the thick line) or foot (below) matching the colour of the number. If the participants made a mistake, they were told to correct the mistake and proceed, while the clock was still ticking. Stage 1 and stage 2 were unilateral. Stage 1 had no crossing over the vertical midline, stage 2 was with crossing over the vertical midline, over the vertical midline, and stage 3 was bilateral. Each child was given three attempts at each stage and the best (shortest) times at each stage were summed to provide a combined score of stages $1-3$.

\section{Player load}

The activity profile during training was measured using a portable global positioning system (GPS) and accelerometer device (MinimaxX S4, Catapult Innovations, Canberra, Australia). The GPS units were placed into the manufacturer-designed harness, which was worn during training. After recording, the data were downloaded and analysed using proprietary software (Sprint, Catapult Sports, Canberra, Australia). Player load is an estimate of training intensity combining the instantaneous rate of change in acceleration in three planes-forward (fwd), sideways (side) and upwards (up)-using the formula:

$$
\sqrt{\left(\begin{array}{c}
\left(f w d_{t=i+1}-f w d_{t=1}\right)^{2}+\left(s i d e_{t=i+1}-s i d e_{t=1}\right)^{2} \\
+\left(u p_{t=i}+1-u p_{t=1}\right)^{2}
\end{array}\right)}
$$

Player load seems to be highly correlated with Edwards' and session-rating of perceived exertion (RPE) methods ${ }^{37}$ and has shown high reliability, suggesting that accelerometers may be used to determine the physical demands of various types of high-intensity training. ${ }^{38}$ The percentage of training time with player load values of $0-1,1-2$ and $>2$, as well as mean values was obtained from the software. ${ }^{38}$

\section{Statistics}

Data are presented as means $( \pm S D)$ unless otherwise stated. Baseline values for the three intervention groups (CON, SSG and CST) were compared using one-way analysis of variance. To evaluate the intervention-induced effects, change scores from 0 to 10 months for CON, SSG and CST were compared using one-way analyses of variance with SAS Enterprise guide 7.1 (SAS institute Inc, Cary, North Carolina, USA). Only data sets with values pre and post were included in the analysis. Between-group differences in training intensity were tested using one-way analysis of variance. The significance level was set at 0.05 .

\section{RESULTS}

No between-group differences were observed in height, body weight, bone variables, lean mass or functional capacity at baseline (figure 2, tables 1 and 2). During the 10-month intervention period, no differences were observed in height and weight change scores for SSG and CON, whereas CST increased more in height than CON (change scores CST vs CON: $0.38 \mathrm{~cm}$, $95 \%$ CI 0.06 to $0.71 \mathrm{~cm}, \mathrm{p}<0.05$ ) (table 1 ).

\section{Training intensity}

Mean player load was higher $(\mathrm{p}<0.01)$ in the SSG than in the CST group $(0.48( \pm 0.15)$ vs $0.34( \pm 0.09))$. The proportional time spent in the highest player load zones was not different between SSG and CST groups (zones $>2$ : $3.2( \pm 2.7) \%$ vs 2.1 $( \pm 1.5) \% ; p>0.05)$, whereas more time was spent in the SSG group in the medium player load zone (zone 1-2: 11.9 $( \pm 5.9) \%$ vs $6.3( \pm 2.2) \% ; \mathrm{p}<0.01)$ and less time in the lowest player load zone (zone 0-1: $84.9( \pm 7.5) \%$ vs $91.4( \pm 3.5) \%$; $\mathrm{p}<0.01)$ compared with the CST group.

\section{Intervention effects on BMD and bone mass}

During the 10-month intervention period, the SSG and CST groups improved whole-body aBMD (change scores SSG vs CON: $8 \mathrm{mg} / \mathrm{cm}^{2}, 95 \%$ CI 3 to $13 \mathrm{mg} / \mathrm{cm}^{2}, \mathrm{p}=0.001$; CST vs CON: $7 \mathrm{mg} / \mathrm{cm}^{2}, 95 \%$ CI 2 to $\left.13 \mathrm{mg} / \mathrm{cm}^{2}, \mathrm{p}=0.005\right)$ and leg BMC (change scores SSG vs CON: $11 \mathrm{~g}, 95 \% \mathrm{CI} 4$ to $18 \mathrm{~g}$, $\mathrm{p}=0.002$; CST vs CON: $11 \mathrm{~g}, 95 \%$ CI 3 to $18 \mathrm{~g}, \mathrm{p}=0.004)$ (figure 2). From 0-10 months, SSG had higher change scores in leg aBMD compared with CON as well as CST (SSG vs CON: $19 \mathrm{mg} / \mathrm{cm}^{2}, 95 \%$ CI 11 to $39 \mathrm{mg} / \mathrm{cm}^{2}, \mathrm{p}<0.001$; SSG vs CST: $12 \mathrm{mg} / \mathrm{cm}^{2}, 95 \%$ CI 3 to $21 \mathrm{mg} / \mathrm{cm}^{2}, \mathrm{p}=0.017$ ), and CST had higher change scores in whole-body BMC compared with CON (CST vs CON: $25 \mathrm{~g}, 95 \%$ CI 10 to $39 \mathrm{~g}, \mathrm{p}<0.01$ ) (figure 2).

\section{Intervention effects on muscular fitness}

The SSG and CST groups had a reduction in number of falls in comparison with CON (change scores SSG vs CON: 2.4 falls/ 

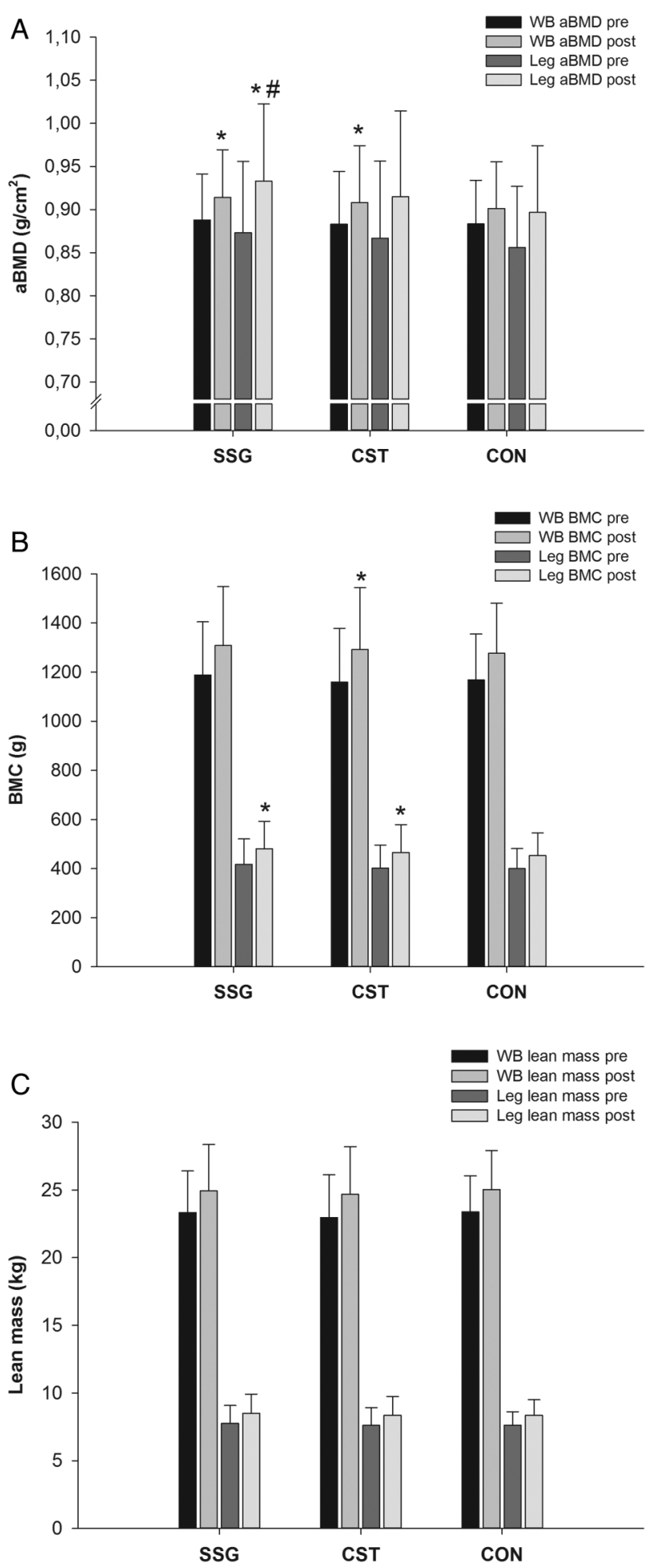

Figure 2 Pre and 10-month values of leg and whole-body BMD (A), bone mass (B) and lean mass (C) for CON and the two intervention groups performing $3 \times 40 \mathrm{~min} /$ week of SSG and CST. Data are presented as mean $( \pm S D)$. * Denotes significant differences in change scores compared with CON, $\mathrm{p}<0.05$. \#Denotes significant differences in change scores compared with CST, $\mathrm{p}<0.05$. aBMD, areal bone mineral density; BMC, bone mineral content; CON, control group; CST, circuit strength training; SSG, small-sided ball games; WB, whole body.

min, $95 \%$ CI 0.3 to 4.5 falls $/ \mathrm{min}, \mathrm{p}=0.03$; CST vs CON: 3.6 falls/min, 95\% CI 1.3 to 5.9 falls/min, $\mathrm{p}=0.003$ ) (table 2). Both training groups also increased jump length in comparison with
CON (SSG vs CON: $10 \%, 95 \%$ CI $5 \%$ to $16 \%, \mathrm{p}<0.001$; CST vs $\mathrm{CON}$ : $9 \%, 95 \%$ CI $3 \%$ to $15 \%, \mathrm{p}=0.002$ ) (table 2 ). No between-group differences ( $p>0.05$ ) were observed in change scores for $20 \mathrm{~m}$ sprint performance, coordination ability or LBM (table 2).

\section{DISCUSSION}

The main findings of the present large-scale RCT were that school-based training interventions with small-sided ball game and circuit strength training improved bone mineralisation, jump performance and postural balance over a 10 -month period in school children aged 8-10 years, suggesting that high-impact PE sessions can be used to promote musculoskeletal health in young children.

Osteoporosis is an age-related chronic skeletal disease characterised by low bone strength due to reduced bone mass and an impairment of bone microarchitecture, and it is associated with increased morbidity and mortality owing to fractures and their complications. ${ }^{39}$ Early prevention of osteoporosis is essential, including the optimisation of peak bone mass, which is attained in the third decade of life. ${ }^{29} 40$ As the optimal time for bone mineral accrual is prepuberty and early puberty, ${ }^{6}$ any osteogenic physical activity at this age is likely to be important to prevent osteoporosis in later life. Some long-term school-based intervention studies involving physical activity have shown no effect on bone health. These include studies involving low-volume $\mathrm{CST}^{41}{ }^{42}$ with low ground reaction forces $(3.5-5$ times body weight) and also interventions with a threefold higher number of PE lessons than normally. ${ }^{15}$ However, it should be emphasised that the latter study was not a randomised trial, and the type and intensity of the physical activity were neither described nor controlled for. Other studies have revealed an osteogenic training effect on BMC and/or density in prepubertal children. ${ }^{29}$ Osteogenic effects were seen in a study using a training programme comparable to that of MacKelvie et $a l^{41}{ }^{42}$ but with higher ground reaction forces reaching eight times the body weight, ${ }^{43}$ and osteogenic effects have also been reported by Meyer et $a l^{14}$ and Detter et $a l^{44}$ using general PE with frequent use of demanding jumping activities.

In the present study, $3 \times 40 \mathrm{~min} /$ week of small-sided ball games elevated whole-body aBMD as well as leg aBMD and BMC, while the same dose of CST induced positive effects on whole-body aBMD along with increases in whole-body and leg BMC. Overall, the magnitude of the osteogenic change was similar to that found in the few other school-based training studies inducing positive osteogenic health effects, ${ }^{29}$ implicating that $3 \times 40 \mathrm{~min} /$ week of SSG or CST is as effective as osteogenic training with other investigated exercise concepts including plyometric training and gymnastics. The intensity recordings during training confirmed that the player load and, thereby, impact were frequently very high during both types of training. They also showed that the average player load and the time spent in medium intensity (zone 1-2) were higher for SSG than for CST, whereas no difference was observed in the time spent in the highest player load zone. These findings may indicate that the time spent with the highest player load is most important for the training adaptations of medium-volume high-intensity training in school children aged 8-10 years. Interestingly, the increase in leg aBMD was significantly higher for ball game group compared with the CST group, indicating that the smallsided games had higher involvement of the lower limbs than the mixed leg and upper body exercises included in the CST protocol. 
Table 2 Functional capacity measurements for the control group (CON) and the two intervention groups performing $3 \times 40 \mathrm{~min} /$ week of small-sided ball games (SSG) and circuit strength training (CST) before (pre) and after (post) the 10-month intervention period

\begin{tabular}{|c|c|c|c|c|c|c|}
\hline & \multicolumn{2}{|l|}{ SSG } & \multicolumn{2}{|l|}{ CST } & \multicolumn{2}{|l|}{ CON } \\
\hline & Pre & Post & Pre & 10 months & Pre & 10 months \\
\hline Balance (falls/min) & $19.2( \pm 8.2)$ & $16.8( \pm 8.0)^{*}$ & $19.6( \pm 8.0)$ & $15.9( \pm 7.7)^{*}$ & $20.8( \pm 8.5)$ & $20.7( \pm 7.7)$ \\
\hline Jump distance (\% of pre) & 100 & $110( \pm 15)^{*}$ & 100 & $109( \pm 18)^{*}$ & 100 & $100( \pm 12)$ \\
\hline 20 m sprint (s) & $4.37( \pm 0.42)$ & $4.24( \pm 0.39)$ & $4.39( \pm 0.33)$ & $4.19( \pm 0.40)$ & $4.41( \pm 0.34)$ & $4.31( \pm 0.33)$ \\
\hline Coordination (s) & $63( \pm 14)$ & $56( \pm 11)$ & $69( \pm 17)$ & $57( \pm 12)$ & $67( \pm 16)$ & $57( \pm 11)$ \\
\hline
\end{tabular}

Data are presented as mean $( \pm \mathrm{SD})$.

*Denotes difference in change scores compared with CON, $\mathrm{p}<0.05$.

In long-term training studies for children, it is always important to consider the role of sexual maturity and growth and to examine whether between-group changes in bone mineralisation are related to the training regimes or to differences in maturity and growth. From the Tanner stage classifications, anthropometrical measurements and body composition results presented in table 1 , it is clear that there were no differences in Tanner results or change scores for height and weight when comparing the ball game group with the control group. On the other hand, the circuit training group had a $0.4 \mathrm{~cm}$ greater change score in height than the control group, and the fraction of girls with changed Tanner scores from stages 1 to 2 was higher in the CST group (36\%) compared with the control group (20\%) and the ball game group (19\%) (table 1). Together, these results provide strong support that the observed osteogenic effects of SSGs are related to the training itself rather than sexual maturity and growth. With regard to the osteogenic effects in the circuit training group, it cannot be ruled out that the positive effects were potentiated by sexual maturity and growth. However, as the osteogenic effects of circuit training compared with control were also significantly greater for the boys, despite no differences in change scores for height and weight for the boys in the two groups and with all the boys in the two groups staying at Tanner stage 1 throughout the intervention period, it is likely that at least part of the observed differences in osteogenic response between the circuit training group and the control group is due to the training regime.

Another interesting observation of the present study was that the intervention effects on bone variables were homogenous among the four schools using SSG and consistently higher than in the two control schools, with change scores ranging from 25 to $27 \mathrm{mg} / \mathrm{cm}^{2}\left(27 \pm 21,25 \pm 11,27 \pm 11,27 \pm 16 \mathrm{mg} / \mathrm{cm}^{2}\right)$ for whole-body aBMD and 53 to $67 \mathrm{mg} / \mathrm{cm}^{2}(59 \pm 35,53 \pm 24$, $64 \pm 23,67 \pm 32 \mathrm{mg} / \mathrm{cm}^{2}$ ) for leg BMD with ball games compared with $16-20 \mathrm{mg} / \mathrm{cm}^{2}\left(20 \pm 15,16 \pm 18 \mathrm{mg} / \mathrm{cm}^{2}\right)$ and $40-41 \mathrm{mg} / \mathrm{cm}^{2}\left(40 \pm 36,41 \pm 22 \mathrm{mg} / \mathrm{cm}^{2}\right)$ for controls. The bone effects for CST were more heterogenic from school to school

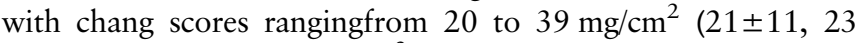
$\left.\pm 12,39 \pm 43,20 \pm 21 \mathrm{mg} / \mathrm{cm}^{2}\right)$ for whole-body aBMD and 32 to $69 \mathrm{mg} / \mathrm{cm}^{2}\left(43 \pm 15,32 \pm 38,69 \pm 51,46 \pm 34 \mathrm{mg} / \mathrm{cm}^{2}\right)$ for leg BMD. These observations suggest that the relatively easy task of delivering small-sided ball game training that elicits high exercise intensity and high involvement of all participants ${ }^{20} 21$ can be done by many different instructors and that the bone effects of the ball game intervention can be achieved in the capital region as well as in the countryside regions, thereby increasing the generalisation of the study results.

In the present study, we also observed a $9 \%$ increase in jump length in both training groups in comparison with the control group, which is similar to the training-induced effects on muscle function in prepubertal children after a 7-week high-intensity interval training (HIIT) programme $\mathrm{e}^{28}$ and a plyometric training programme conducted in elementary schools. ${ }^{27}$ However, the effects on muscle strength were greater than improvements observed in other school-based interventions involving enhanced general physical activity. ${ }^{45}$ Thus, in the study by Löfgren et al, ${ }^{45}$ no jump performance effects were observed in boys after 2 years of extra PE lessons, whereas positive effects were observed in vertical jump height in the investigated girls. Despite the high intensity, we did not observe any significant effects on either gross motor skill or $20 \mathrm{~m}$ sprint time, even though plyometric training has previously been shown to improve sprint performance after just 11 weeks of training. ${ }^{46}$

Last, but not least, we observed that both training types resulted in an enhanced postural balance. Only a few studies have investigated the effects of physical activity interventions on postural balance in healthy children. A Czech study found no significant effects of a 10 -week climbing programme on postural balance, ${ }^{47}$ while a Canadian study of specific balance training using Nintendo Wii or more traditional exercises showed considerable improvements in postural balance of $26 \%$ and $23 \%$, respectively. ${ }^{48}$ Overall, the present findings show that the chosen types of high-intensity training, that is, SSG as well as CST, had broad-spectrum effects on musculoskeletal health with an elevated bone mineralisation, improved muscle strength and an enhanced postural balance, and that both types of training have combined positive musculoskeletal effects that may have a preventive effect on the future risk of falls and fractures in later life.

A major advantage of the present study, apart from the cluster-randomised controlled design with long-term training, was that the exercise interventions were well controlled throughout the intervention period, making it possible to conclude specifically that medium-volume SSG and CST induced a sufficient musculoskeletal load to enhance bone formation, postural balance and jumping performance. Another strength of the present study is that sexual maturity and growth were measured with prepost Tanner classifications for all children by the same medical doctor and state-of-the-art prepost measurements of height, weight and body composition. However, it should be acknowledged as a limitation that the number of participants was not sufficiently high to distinguish between the training-induced effects for girls versus boys or for sports club active versus non-sports club active children and that the control participants, although high in numbers, only came from one urban school and one rural school. Moreover, it is considered a limitation that the overall physical activity of the participants was not determined before and during the 10-month intervention period, but solely the exercise intensity and the physical demands of the guided sessions with SSG and CST. 
In summary, the present study is, as far as we know, the first RCT to investigate the musculoskeletal effects of a school-based intervention involving small-sided ball games and circuit strength training for children aged 8-10 years, and the current results demonstrate that significant structural and functional musculoskeletal adaptations can be achieved in a well-controlled school-based setting. The observations confirm that, in childhood, the skeleton adapts to the physiological changes induced by physical training and suggest that compared with other physiological training modalities, HIIT and odd-impact training may be a favoured intervention for achieving musculoskeletal changes. This may have beneficial consequences for long-term osteogenic health.

\section{What are the findings?}

- Small-sided ball game and circuit strength training for children aged 8-10 years provide high musculoskeletal impact.

- Small-sided ball game and circuit strength training conducted within the school curriculum are suitable for improving musculoskeletal health in young children.

- The positive effects of well-structured medium-volume ball game and circuit strength training on bone mineral accrual are followed by improvements in balance and jumping distance in young children.

\section{How might it impact on clinical practice in the future?}

- Two evidence-based, well-defined, school-based physical activity concepts with positive effects on musculoskeletal health are now developed and tested for young children.

- The FIT FIRST concept, with frequent intense training, can be implemented in the school system for children aged 8-10 years, as well as other settings.

- The small-sided ball game and circuit strength training can be conducted in small areas on a variety of indoor and outdoor surfaces, thereby providing further potential for a large-scale implementation.

Acknowledgements The authors would like to thank the participating pupils, teachers and schools in Frederikssund and Copenhagen for their contribution, as well as Frederikssund Municipality for its collaboration and support. The author would like to thank Henrik Pedersen and Therese Hornstrup for their excellent technical assistance. Also, the author thanks to Jan R. Jacobsen, Henrik Oksholm and Ida Elkjær running most of the training sessions and for their assistance during testing sessions. The Copenhagen Centre for Team Sport and Health is supported by a grant from the Nordea Foundation (Nordea-fonden). In addition, this study was supported by the FIFA Medical Assessment and Research Centre (F-MARC), the Danish Football Association (DBU), the Danish Ministry of Culture (KFU), Aase and Ejnar Danielsens Foundation and Augustinus Fonden. The foundations did not participate in the analysis, interpretation or writing of the paper.

Contributors PK and PRH designed the study. CMN, MNL, PRH, JB and PK described the detailed protocol for ethical approval and for registration in clinical trials. MNL, JB and PK successfully applied for financial support to the study. MNL, CMN, MM and PK carried out the training regime and collected the data. All authors participated in the analyses of the data and its interpretation. MNL and PK drafted the first version of the article and all authors revised the manuscript critically for important intellectual content. All authors approved the final version of the article.

Funding The present study was funded by Nordea-fonden 02-2011-4360, Aase and Ejnar Danielsens Foundation 1047491001, Augustinus Fonden 1042861001,
FIFA Medical Assessment and Research Centre (F-MARC) F-MARC Project 31964, the Danish Football Association (DBU) 31964 and the Danish Ministry of Culture 1045171001.

\section{Competing interests None declared.}

Ethics approval The study was approved by the committees on Biomedical Research Ethics for the Capital Region of Denmark (J.no. H-3-2013-038).

Provenance and peer review Not commissioned; externally peer reviewed.

Data sharing statement As described in the clinical trial registration (NCT02000492), articles dealing with the cardiovascular effects of $3 \times 40 \mathrm{~min} /$ week of small-sided games and circuit strength training will also be published, along with articles evaluating the cardiovascular and musculoskeletal training effects of $5 \times 12 \mathrm{~min} /$ week of small-sided games and interval running.

Open Access This is an Open Access article distributed in accordance with the Creative Commons Attribution Non Commercial (CC BY-NC 4.0) license, which permits others to distribute, remix, adapt, build upon this work non-commercially, and license their derivative works on different terms, provided the original work is properly cited and the use is non-commercial. See: http://creativecommons.org/ licenses/by-nc/4.0/

\section{REFERENCES}

1 Kannus P, Niemi S, Parkkari J, et al. Hip fractures in Finland between 1970 and 1997 and predictions for the future. Lancet 1999;353:802-5

2 Karlsson MK, Nordqvist A, Karlsson C. Physical activity, muscle function, falls and fractures. Food Nutr Res 2008;52:1-7.

3 Khan K. Physical activity and bone health: Human Kinetics. 2001.

4 Sundberg M, Gärdsell P, Johnell O, et al. Physical activity increases bone size in prepubertal boys and bone mass in prepubertal girls: a combined cross-sectional and 3-year longitudinal study. Calcif Tissue Int 2002;71:406-15.

5 Weaver CM, Gordon CM, Janz KF, et al. The National Osteoporosis Foundation's position statement on peak bone mass development and lifestyle factors: a systematic review and implementation recommendations. Osteoporosis Int 2016;27:1281-386

6 Bass S, Pearce G, Bradney M, et al. Exercise before puberty may confer residual benefits in bone density in adulthood: studies in active prepubertal and retired female gymnasts. J Bone Miner Res 1998;13:500-7

7 van Sluijs EM, McMinn AM, Griffin SJ. Effectiveness of interventions to promote physical activity in children and adolescents: systematic review of controlled trials. BMJ 2007:335:703

8 Dobbins $M$, De Corby $K$, Robeson $P$, et al. School-based physical activity programs for promoting physical activity and fitness in children and adolescents aged 6-18. Cochrane Database Syst Rev 2009;(1):CD007651

9 MacKelvie K, Khan K, McKay H. Is there a critical period for bone response to weight-bearing exercise in children and adolescents? A systematic review. $\mathrm{Br} J$ Sports Med 2002;36:250-7.

10 Turner $\mathrm{CH}$, Robling AG. Designing exercise regimens to increase bone strength. Exerc Sport Sci Rev 2003;31:45-50.

11 Linden C, Ahlborg HG, Besjakov J, et al. A school curriculum-based exercise program increases bone mineral accrual and bone size in prepubertal girls: two-year data from the Pediatric Osteoporosis Prevention (POP) study. J Bone Miner Res 2006;21:829-35

12 Lindén $\mathrm{C}$, Alwis $\mathrm{G}$, Ahlborg $\mathrm{H}$, et al. Exercise, bone mass and bone size in prepubertal boys: one-year data from the pediatric osteoporosis prevention study. Scand J Med Sci Sports 2007;17:340-7

13 Hasselstrøm HA, Karlsson MK, Hansen SE, et al. A 3-year physical activity intervention program increases the gain in bone mineral and bone width in prepubertal girls but not boys: the prospective Copenhagen School Child Interventions Study (CoSCIS). Calcif Tissue Int 2008;83:243-50.

14 Meyer U, Romann M, Zahner L, et al. Effect of a general school-based physical activity intervention on bone mineral content and density: a cluster-randomized controlled trial. Bone 2011:48:792-7.

15 Heidemann $M$, Jespersen $E$, Holst $R$, et al. The impact on children's bone health of a school-based physical education program and participation in leisure time sports: the Childhood Health, Activity and Motor Performance School (the CHAMPS) study, Denmark. Prev Med 2013;57:87-91.

16 Krustrup P, Aagaard P, Nybo L, et al. Recreational football as a health promoting activity: a topical review. Scand J Med Sci Sports 2010;20(Suppl 1):1-13.

17 Helge EW, Aagaard P, Jakobsen MD, et al. Recreational football training decreases risk factors for bone fractures in untrained premenopausal women. Scand J Med SCi Sports 2010;20(Suppl 1):31-9.

18 Uth J, Hornstrup T, Christensen JF, et al. Football training in men with prostate cancer undergoing androgen deprivation therapy: activity profile and short-term skeletal and postural balance adaptations. Eur J App/ Physiol 2016:116:471-80.

19 Andersen TR, Schmidt JF, Pedersen MT, et al. The effects of 52 weeks of soccer or resistance training on body composition and muscle function in +65 -year-old healthy males-a randomized controlled trial. PLOS ONE 2016;11:e0148236. 
20 Randers MB, Nybo L, Petersen J, et al. Activity profile and physiological response to football training for untrained males and females, elderly and youngsters: influence of the number of players. Scand J Med Sci Sports 2010;20(Suppl 1):14-23.

21 Bendiksen M, Williams CA, Hornstrup $T$, et al. Heart rate response and fitness effects of various types of physical education for 8- to 9-year-old schoolchildren. Eur J Sport Sci 2014;14:861-9.

22 Ferry B, Duclos M, Burt L, et al. Bone geometry and strength adaptations to physical constraints inherent in different sports: comparison between elite female soccer players and swimmers. J Bone Miner Metab 2011;29:342-51.

23 Vicente-Rodriguez G, Jimenez-Ramirez J, Ara I, et al. Enhanced bone mass and physical fitness in prepubescent footballers. Bone 2003;33:853-9.

24 Seabra A, Marques E, Brito J, et al. Muscle strength and soccer practice as major determinants of bone mineral density in adolescents. Joint Bone Spine 2012;79:403-8.

25 Vicente-Rodriguez G, Ara I, Perez-Gomez J, et al. Muscular development and physical activity as major determinants of femoral bone mass acquisition during growth. Br J Sports Med 2005;39:611-16.

26 Vicente-Rodriguez G, Ara I, Perez-Gomez J, et al. High femoral bone mineral density accretion in prepubertal soccer players. Med Sci Sports Exerc 2004;36: 1789-95.

27 Faigenbaum AD, Farrell AC, Radler T, et al. 'Plyo Play': a novel program of short bouts of moderate and high intensity exercise improves physical fitness in elementary school children. Phys Educ 2009;66:37.

28 Baquet $\mathrm{G}$, Guinhouya $\mathrm{C}$, Dupont $\mathrm{G}$, et al. Effects of a short-term interval training program on physical fitness in prepubertal children. J Strength Cond Res 2004;18:708-13

29 Hind K, Burrows M. Weight-bearing exercise and bone mineral accrual in children and adolescents: a review of controlled trials. Bone 2007;40:14-27.

30 Margulies L, Horlick M, Thornton JC, et al. Reproducibility of pediatric whole body bone and body composition measures by dual-energy X-ray absorptiometry using the GE Lunar Prodigy. J Clin Densitom 2005;8:298-304.

31 Adam C, Klissouras V, Ravazzolo M, et al. EUROFIT: European test of physical fitness. Rome: Council of Europe. Committee for the development of sport, 1988:10-70.

32 Fjortoft I. Motor fitness in pre-primary school children: the EUROFIT motor fitness test explored on 5-7-year-old children. PES 2010;12:424-36.

33 Ortega FB, Artero EG, Ruiz JR, et al. Reliability of health-related physical fitness tests in European adolescents. The HELENA Study. Int J Obes 2008;32:S49-57.
34 Castro-Piñero J, Ortega FB, Artero EG, et al. Assessing muscular strength in youth: usefulness of standing long jump as a general index of muscular fitness. J Strength Cond Res 2010;24:1810-17.

35 Zahner L, Puder JJ, Roth R, et al. A school-based physical activity program to improve health and fitness in children aged 6-13 years ('Kinder-Sportstudie KISS'): study design of a randomized controlled trial [ISRCTN15360785]. BMC Public Health 2006;6:147.

36 Essendrop M, Hansen L, Klausen KJ. Balance and co-ordination abilities related to training in youth athletes. Children and Exercise, Washington Singer Press 1997;XIX:161-6.

37 Casamichana D, Castellano J, Calleja-Gonzalez J, et al. Relationship between indicators of training load in soccer players. J Strength Cond Res 2013;27:369-74.

38 Boyd LJ, Ball K, Aughey RJ. The reliability of MinimaxX accelerometers for measuring physical activity in Australian football. Int I Sports Physiol Perform 2011;6:311-21.

39 Kanis JA, Melton LJ, Christiansen C, et al. The diagnosis of osteoporosis. J Bone Miner Res 1994;9:1137-41.

40 Bachrach LK. Acquisition of optimal bone mass in childhood and adolescence. Trends Endocrinol Metab 2001;12:22-8.

41 MacKelvie KJ, McKay HA, Petit MA, et al. Bone mineral response to a 7-month randomized controlled, school-based jumping intervention in 121 prepubertal boys: associations with ethnicity and body mass index. J Bone Miner Res 2002;17:834-44.

42 MacKelvie KJ, McKay HA, Khan KM, et al. A school-based exercise intervention augments bone mineral accrual in early pubertal girls. J Pediatr 2001;139:501-8.

43 Fuchs RK, Bauer JJ, Snow CM. Jumping improves hip and lumbar spine bone mass in prepubescent children: a randomized controlled trial. J Bone Miner Res 2001;16:148-56.

44 Detter FTL, Rosengren BE, Dencker $\mathrm{M}$, et al. A 5-year exercise program in pre- and peripubertal children improves bone mass and bone size without affecting fracture risk. Calcif Tissue Int 2013;92:385-93.

45 Löfgren B, Daly RM, Nilsson JA, et al. An increase in school-based physical education increases muscle strength in children. Med Sci Sports Exerc 2013;45:997-1003.

46 Chaouachi $A$, Othman $A B$, Hammami $R$, et al. The combination of plyometric and balance training improves sprint and shuttle run performances more often than plyometric-only training with children. J Strength Cond Res 2014;28:401-12.

47 Balas J, Bunc V. Short-term influence of climbing activities on strength, endurance and balance within school physical education. Int J Fit 2007;3:33-42.

48 Sheehan D, Katz $L$. The impact of a six week exergaming curriculum on balance with grade three school children using the Wii FIT (TM). Int J Comput Sci Sport 2012;11:5-22. 